\title{
CMC A-net surfaces in three dimensional Heisenberg group
}

\author{
Gulden Altay Suroglu ${ }^{1}$, Mahmut Ergut ${ }^{2}$ and Talat Korpinar ${ }^{3}$ \\ ${ }^{1}$ Department of Mathematics, Firat University, Elazig, Turkey \\ ${ }^{2}$ Department of Mathematics, Namik Kemal University, Tekirdag, Turkey \\ ${ }^{2}$ Department of Mathematics, Mus Alparslan University, Mus, Turkey
}

Received: 19 December 2016, Accepted: 22 July 2017

Published online: 5 March 2018.

\begin{abstract}
In this paper, we obtain the mean curvature of a A- net surface in three dimensional Heisenberg group $\mathbb{H}_{3}$. Moreover, we give some characterizations of this surface according to Levi- Civita connections of $\mathbb{H}_{3}$. Using the mean curvature, a new characterization for the cmc A- net surface. Finally, we draw cmc A- net surface by Mathematica.
\end{abstract}

Keywords: Heisenberg group, cmc surface, A- net surface.

\section{Introduction}

The study of mean curvature expands back to 18. century. Later, Lagrange looked for a necessary condition to minimizing a certain integral then, he found the minimal surface equation. Meusnier firstly define the term of mean curvature. Then, important mathematicians such as Gauss and Weierstrass devoted much of their studies to these surfaces. Finally, constant mean curvature surfaces remain somewhat mysterious to this day.

The significance of CMC hypersurfaces as useful tools for studying the physics of general relativistic spacetimes is widely recognized. Nevertheless little is yet known about the class of spacetimes which admit them. It has been conjectured that every maximally extended, globally hyperbolic, spatially compact solution of Einstein's equations (in vacuum or with "reasonable" source field coupling) can be foliated by CMC hypersurfaces. However this conjecture is known to be true only for a handful of examples such as the spatially homogeneous cosmological models, [7]. Also, The partial differential equation $H=$ const. can be considered the Euler equation to the variational problem.

In [16], Soyuçok interested with the Bonnet problem of determining the surfaces in Euclidean three dimensional space which can admit at least one nontrivial isometry that preserves principal curvatures. This problem considered locally and examined the general case. Then, In [8], Kanbay considered the Bonnet ruled surfaces which admit only one non-trivial isometry that preserves the principal curvatures, then, she determined the Bonnet ruled surfaces whose generators and orthogonal trajectories form a special net called an A-net.

In [4], Brander studied constant positive Gauss curvature $K$ surfaces in the 3 -sphere $S^{3}$ with $0<K<1$ as well as constant negative curvature surfaces. They showed that the so-called normal Gauss map for a surface in $S^{3}$ with Gauss curvature $K<1$ is Lorentz harmonic with respect to the metric induced by the second fundamental form if and only if $K$ is constant. They gave a uniform loop group formulation for all such surfaces with $K \neq 0$, and use the generalized d'Alembert method to construct examples. Then, they obtain that those representation gives a natural correspondence 
between such surfaces with $K<0$ and those with $0<K<1$. In [6], Galvez deal with some classical results on complete surfaces with constant Gauss curvature in 3-dimensional space forms with using a modern approach. Then, he obtain characterizetion on the complete surfaces with positive constant Gauss curvature in $S^{3}, \mathbb{R}^{3}$ or $H^{3}$ and proved the Liebmann theorem and show that the only complete examples must be totally umbilical round spheres. He deduce that there is no complete surface in $S^{3}$ with constant Gauss curvature $K(I) \in(0,1)$. In [12], Lopez studied a parabolic surface in hyperbolic space $H^{3}$ is a surface invariant by a group of parabolic isometries, then, describe all parabolic surfaces with constant Gaussian curvature. In [13], Lopez gave a space-like or time-like surface in Lorentz-Minkowski three-space $L^{3}$ generated by a oneparameter family of circular arcs and later, he obtain if the Gauss curvature $K$ is a nonzero constant, then $M$ is a surface of revolution. We also describe the parametrizations for $M$ when $K \equiv 0$.

In this paper, we studied A- net surfaces in three dimensional Heisenberg group. Then, we made some characterizations of curvatures of these surfaces.

\section{Heisenberg Group $\mathbb{H}_{3}$}

The Heisenberg group historically originates in and still has its strongest ties to quantum physics: there it is a group of unitary operators acting on the space of states induced from those observables on a linear phase space, which are given by linear or by constant functions. So any Heisenberg group is a subgroup of a group of observables in certain simple examples of quantum mechanical systems.

The Heisenberg group $\mathbb{H}_{3}$ is defined as $\mathbb{R}^{3}$ with the group operation

$$
(x, y, z) *\left(x_{1}, y_{1}, z_{1}\right)=\left(x+x_{1}, y+y_{1}, z+z_{1}+\frac{1}{2}\left(x y_{1}-x_{1} y\right)\right) .
$$

The left-invariant Riemannain metric on $\mathbb{H}_{3}$ is given by

$$
g=d s^{2}=d x^{2}+d y^{2}+(x d y+d z)^{2} .
$$

The left invariant orthonormal frame on $\mathbb{H}_{3}$, which is belong to Riemannian metric $g$

$$
\mathbf{e}_{1}=\frac{\partial}{\partial x}, \mathbf{e}_{2}=\frac{\partial}{\partial y}+x \frac{\partial}{\partial z}, \mathbf{e}_{3}=\frac{\partial}{\partial z} .
$$

For the covariant derivatives of the Levi-Civita connection of the left-invariant metric $g$,

$$
\nabla_{\mathbf{e}_{i}} \mathbf{e}_{j}=\frac{1}{2}\left[\begin{array}{ccc}
0 & \mathbf{e}_{3} & -\mathbf{e}_{2} \\
-\mathbf{e}_{3} & 0 & \mathbf{e}_{1} \\
-\mathbf{e}_{2} & \mathbf{e}_{1} & 0
\end{array}\right],
$$

where the $(i, j)$-element in the table above equals $\nabla_{\mathbf{e}_{i}} \mathbf{e}_{j}$ for our basis. Also, we have the Heisenberg bracket relations

$$
\left[\mathbf{e}_{1}, \mathbf{e}_{2}\right]=\mathbf{e}_{3},\left[\mathbf{e}_{1}, \mathbf{e}_{3}\right]=\left[\mathbf{e}_{2}, \mathbf{e}_{3}\right]=0 .
$$

\section{A- net Surfaces with Constant Gaussian in $\mathbb{H}_{3}$}

In this chapter, we characterized A- net surfaces in three dimensional Heisenberg group. Then, we obtain constant Gaussian curvature of this surface. A A-net on a surface such that, when this net is parametrized, the conditions

$$
E=G, F=0, h_{12}=\text { constant } \neq 0, h_{21}=\text { constant } \neq 0
$$


are satisfied, is called an A-net, where $E, F, G$ are the coefficients of the first fundamental form of the surface and $h_{11}$, $h_{12}, h_{21}, h_{22}$ are the coefficients of the second fundamental form.

Let

$$
\varphi(x, y)=\mu(x) \mathbf{e}_{1}+\xi(y) \mathbf{e}_{2}+\rho(x, y) \mathbf{e}_{3}
$$

be a surface in $\left(\mathbb{H}_{3}, g\right)$. If we take derivatives of the surface, which is given with the parametrization (1), we have

$$
\begin{aligned}
& \varphi_{x}(x, y)=\mu^{\prime}(x) \mathbf{e}_{1}+\rho_{x}(x, y) \mathbf{e}_{3}, \\
& \varphi_{y}(x, y)=\xi^{\prime}(y) \mathbf{e}_{2}+\rho_{y}(x, y) \mathbf{e}_{3} .
\end{aligned}
$$

Then, components of the first fundamental form of the surface are

$$
\begin{aligned}
& E=\rho_{x}^{2}+\mu^{\prime 2}(x), \\
& F=\rho_{x} \rho_{y} \\
& G=\xi^{\prime 2}(y)+\rho_{y}^{2} .
\end{aligned}
$$

So, if $F=0$, from equations (3) we have

$$
\rho_{y}=0 \text { or } \rho_{x}=0 .
$$

From (4), if $\rho_{y}=0$, then $\rho(x, y)=\alpha(x)$. If $\rho_{x}=0$, then $\rho(x, y)=\beta(y)$.

Theorem 1. Let $\varphi(x, y)$ be a surface which is parameterized as (1). If $\rho_{y}=0$, then the mean curvature of the surface $\varphi(x, y)$

$$
H=\frac{\mu^{\prime}(x)}{\left(\alpha^{\prime}(x)+\mu^{\prime}(x)\right)^{3 / 2}}\left(-\mu^{\prime \prime}(x) \alpha^{\prime}(x)+\mu^{\prime}(x) \alpha^{\prime \prime}(x)\right) .
$$

Proof. From equations (2), we have

$$
\begin{aligned}
& \mathbf{E}_{1}=\mu^{\prime}(x) \mathbf{e}_{1}+\alpha^{\prime}(x) \mathbf{e}_{3}, \\
& \mathbf{E}_{2}=\xi^{\prime}(y) \mathbf{e}_{2} .
\end{aligned}
$$

Then, components of the first fundamental form of the surface $\varphi(x, y)$ are

$$
\begin{aligned}
& E=\alpha^{\prime 2}(x)+\mu^{\prime 2}(x), \\
& F=0, \\
& G=\xi^{\prime 2}(y) .
\end{aligned}
$$

So, the induced metric is

$$
\tilde{g}=\left(\alpha^{\prime}(x)+\mu^{\prime 2}(x)\right) d x^{2}+\xi^{\prime 2}(y) d y^{2} .
$$

The unit normal vector field of the surface is

$$
\mathbf{N}=\frac{1}{\sqrt{\mu^{\prime 2}(x)+\alpha^{\prime}(x)^{2}}}\left(-\alpha^{\prime}(x) \mathbf{e}_{1}+\mu^{\prime}(x) \mathbf{e}_{3}\right) .
$$


Then, we have

$$
\begin{aligned}
\nabla_{\mathbf{E}_{1}} \mathbf{E}_{1} & =\mu^{\prime}(x)\left(\mu^{\prime \prime}(x) \mathbf{e}_{1}-\alpha^{\prime}(x) \mathbf{e}_{2}+\alpha^{\prime \prime}(x) \mathbf{e}_{3}\right) \\
\nabla_{\mathbf{E}_{1}} \mathbf{E}_{2} & =\frac{\xi^{\prime}(y)}{2}\left\{\alpha^{\prime}(x) \mathbf{e}_{1}+\mu^{\prime}(x) \mathbf{e}_{3}\right\}, \\
\nabla_{\mathbf{E}_{2}} \mathbf{E}_{1} & =\frac{\xi^{\prime}(y)}{2}\left\{\alpha^{\prime}(x) \mathbf{e}_{1}-\mu^{\prime}(x) \mathbf{e}_{3}\right\}, \\
\nabla_{\mathbf{E}_{2}} \mathbf{E}_{2} & =\xi^{\prime}(y) \xi^{\prime \prime}(y) \mathbf{e}_{2} .
\end{aligned}
$$

So, from (7) and (8)-(11) equations, components of the second fundamental form of the surface are

$$
\begin{aligned}
& h_{11}=\frac{\mu^{\prime}(x)}{\sqrt{\mu^{\prime 2}(x)+\alpha^{\prime}(x)^{2}}}\left(\mu^{\prime}(x) \alpha^{\prime \prime}(x)-\mu^{\prime \prime}(x) \alpha^{\prime}(x)\right), \\
& h_{12}=\frac{\xi^{\prime}(y)}{2 \sqrt{\mu^{\prime 2}(x)+\alpha^{\prime}(x)^{2}}}\left(\mu^{\prime 2}(x)-\alpha^{\prime}(x)^{2}\right), \\
& h_{21}=-\frac{1}{2} \xi^{\prime}(y) \sqrt{\mu^{\prime 2}(x)+\alpha^{\prime}(x)^{2}}, \\
& h_{22}=0 .
\end{aligned}
$$

Then, the mean curvature of the surface $\varphi(x, y)$

$$
H=\frac{\mu^{\prime}(x)}{\left(\alpha^{\prime}(x)+\mu^{\prime}(x)\right)^{3 / 2}}\left(-\mu^{\prime \prime}(x) \alpha^{\prime}(x)+\mu^{\prime}(x) \alpha^{\prime \prime}(x)\right) .
$$

Theorem 2. Let $\varphi(x, y)$ be a surface which is parameterized as (1). If $\varphi(x, y)$ is an A-net cmc surface with $\rho_{y}=0$, then

$$
\varphi(x, y)=\mu(x) \mathbf{e}_{1}+\xi(y) \mathbf{e}_{2}+\left( \pm \frac{C^{3 / 2} c_{1} \sqrt{-x^{2}-2 x c_{2}-c_{2}^{2}}}{-1+2 C^{2} x c_{1}^{2} c_{2}+2 C^{2} c_{1}^{2} c_{2}^{2}}\right) \mathbf{e}_{3} .
$$

Proof. From (13), (14), we have

$$
h_{12}=\text { constant }=A \Longrightarrow \frac{\xi^{\prime}(y)}{2 \sqrt{\mu^{\prime 2}(x)+\alpha^{\prime}(x)^{2}}}\left(\mu^{\prime 2}(x)-\alpha^{\prime}(x)^{2}\right)=A
$$

and

$$
h_{21}=\text { constant }=B \Longrightarrow-\frac{1}{2} \xi^{\prime}(y) \sqrt{\mu^{\prime 2}(x)+\alpha^{\prime}(x)^{2}}=B .
$$

From (18), (19), we have

$$
\mu^{\prime}(x)=\sqrt{\alpha^{\prime}(x)^{2}+C}
$$

where $C=\frac{A}{B}$. Then, from (18)- (22), the mean curvature is

$$
H=\frac{C \alpha^{\prime \prime}(x)}{\left(2 \alpha^{\prime}(x)^{2}+C\right)^{3 / 2}}
$$


So, if the mean curvature of the surface is a constant, from (21), we have

$$
\frac{C \alpha^{\prime \prime}(x)}{\left(2 \alpha^{\prime}(x)^{2}+C\right)^{3 / 2}}=D \text {. }
$$

Derivative of the equation (22), we have

$$
\frac{C\left(\alpha^{\prime \prime \prime}(x)\left(C+2 \alpha^{\prime 2}(x)\right)-6 \alpha^{\prime \prime 2}(x) \alpha^{\prime}(x)\right)}{\left(2 \alpha^{\prime}(x)^{2}+C\right)^{5 / 2}}=0
$$

So, from equation (23),

$$
C\left(\alpha^{\prime \prime \prime}(x)\left(C+2 \alpha^{\prime 2}(x)\right)-6 \alpha^{\prime \prime 2}(x) \alpha^{\prime}(x)\right)=0 .
$$

In this situation, we have two possibilies.

(i) If $C=0$, then we have $h_{12}=0$, which is conrtadicts with the surface is a A- net surface.

(ii) Let

$$
\alpha^{\prime \prime \prime}(x)\left(C+2 \alpha^{\prime 2}(x)\right)-6 \alpha^{\prime \prime 2}(x) \alpha^{\prime}(x)=0 .
$$

Then, if we notice that

$$
\alpha^{\prime}(x)=P(x)
$$

the equation (25) become

$$
P^{\prime \prime}(x)\left(C+2 P^{2}(x)\right)-6 P^{\prime 2}(x) P(x)=0 .
$$

So, we have

$$
P(x)= \pm \frac{C^{3 / 2} c_{1} \sqrt{-x^{2}-2 x c_{2}-c_{2}^{2}}}{\sqrt{-1+2 C^{2} x c_{1}^{2} c_{2}+2 C^{2} c_{1}^{2} c_{2}^{2}}} .
$$

With integrate of the equation (27) according to $x$, we have

$$
\alpha(x)= \pm \frac{\sqrt{-x^{2}-2 x c_{2}-c_{2}^{2}} \sqrt{-1+2 C^{2} c_{1}^{2}\left(x+c_{2}\right)^{2}}}{2 c_{1} \sqrt{C}\left(x+c_{2}\right)}
$$

where $c_{1}$ and $c_{2}$ are integration constant. So, if $\varphi(x, y)$ is a cmc A- net surface, we have

$$
\varphi(x, y)=\mu(x) \mathbf{e}_{1}+\xi(y) \mathbf{e}_{2}+\left( \pm \frac{\sqrt{-x^{2}-2 x c_{2}-c_{2}^{2}} \sqrt{-1+2 C^{2} c_{1}^{2}\left(x+c_{2}\right)^{2}}}{2 c_{1} \sqrt{C}\left(x+c_{2}\right)}\right) \mathbf{e}_{3} .
$$

So, the proof is complete.

Corollary 1. Let $\varphi(x, y)$ be A- net surface which is parameterized as (1) with $\rho_{y}=0$. Then, if $\alpha(x)=c_{3} x+c_{4}$, where $c_{3}$ and $c_{4}$ are integration constant, then the Gaussian curvature of the $\varphi(x, y)$ is a non-zero constant.

Proof. From equations (6), (12)-(15), the Gaussian curvature is

$$
K=-\frac{A B}{\sqrt{2 \alpha^{\prime}(x)^{2}+C}} .
$$

So, if

$$
\alpha(x)=c_{3} x+c_{4},
$$

$\varphi(x, y)$ has constant Gaussian curvature. 
Theorem 3. Let $\varphi(x, y)$ be a surface which is parameterized as (1). If $\rho_{x}=0$, then the mean curvature of the surface $\varphi(x, y)$

$$
H=\frac{\xi^{\prime}(y)}{\left(\alpha^{\prime}(x)+\xi^{\prime}(y)\right)^{3 / 2}}\left(-\xi^{\prime \prime}(y) \alpha^{\prime}(x)+\xi^{\prime}(y) \alpha^{\prime \prime}(x)\right) .
$$

Proof. The proof obtains like Theorem 2.

Corollary 2. Let $\varphi(x, y)$ be a surface which is parameterized as (1). If $\varphi(x, y)$ is an A- net cmc surface with $\rho_{x}=0$, then

$$
\varphi(x, y)=\mu(x) \mathbf{e}_{1}+\xi(y) \mathbf{e}_{2}+\left( \pm \frac{C^{3 / 2} c_{1} \sqrt{-y^{2}-2 y c_{2}-c_{2}^{2}}}{-1+2 C^{2} y c_{1}^{2} c_{2}+2 C^{2} c_{1}^{2} c_{2}^{2}}\right) \mathbf{e}_{3}
$$

Corollary 3. Let $\varphi(x, y)$ be A- net surface which is parameterized as (1) with $\rho_{x}=0$. Then, the Gaussian curvature of the $\varphi(x, y)$ is a non-zero constant if

$$
\alpha(y)=c_{3} y+c_{4}
$$

Example 1. Let $\varphi(x, y)$ be a surface in $\left(\mathbb{H}_{3}, g\right)$ which is parameterized as

$$
\varphi(x, y)=\left(\cos x, \sin y, \cos x-\frac{C^{3 / 2} c_{1} \sqrt{-x^{2}-2 x c_{2}-c_{2}^{2}}}{-1+2 C^{2} x c_{1}^{2} c_{2}+2 C^{2} c_{1}^{2} c_{2}^{2}}\right) .
$$

This surface is a A- net cmc surface in $\left(\mathbb{H}_{3}, g\right)$.

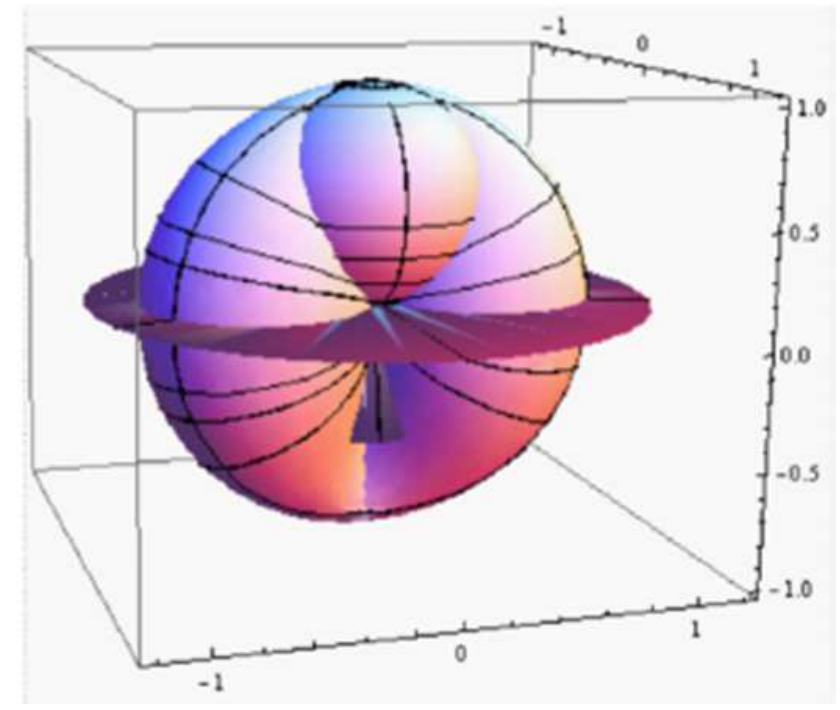

Fig. 1: A-net cmc surface in $\left(\mathbb{H}_{3}, g\right)$.

\section{Competing interests}

The authors declare that they have no competing interests.

\section{Authors' contributions}

All authors have contributed to all parts of the article. All authors read and approved the final manuscript. 


\section{References}

[1] J. Aledo, M. Espinar, J. Galvez, Timelike Surfaces in the Lorentz-Minkowski Space with Prescribed Gaussian Curvature and Gauss Map, Journal of Geometry and Physics., 56 (2006), 1357- 1369.

[2] G. Altay, H. Öztekin, Translation Surfaces Generated by Mannheim Curves in Three Dimensional Euclidean Space, Gen. Math. Notes, 26 (2015), 28-34.

[3] A. Bobenko, U. Either, Bonnet Surfaces and Painleve Equations, J. Reine Angew Math., 499 (1998), 47- 79.

[4] D. Brander, J. Inoguchi, S. Kobayashi, Constant Gaussian Curvature Surfaces in The 3- Sphere Via Loop Groups, ArXiv: 1301.5999v1, (2013). [

[5] R. Caddeo, P. Piu, A. Ratto, Rotational Surfaces in $\mathrm{H}_{3}$ with Constant Gauss Curvature,

[6] J. Galvez, Surfaces of constant curvature in 3-dimensional space forms, Matematica Contemporanea, 37 (2009), 01-42

[7] J. Isenberg and V. Moncrief, The Existence of Constant Mean Curvature Foliations of Gowdy 3-Torus Spacetimes, Commun. Math. Phys. 86 (1982), 485-493.

[8] F. Kanbay, Bonnet Ruled Surfaces, Acta Mathematica Sinica, English Series, 21 (2005), 623- 630.

[9] T. Körpınar, E. Turhan, Spacelike Biharmonic New Type B-Slant Helices According to Bishop Frame in the Lorentzian Heisenberg Group $H^{3}$, Gen. Math. Notes, 10 (2012), 36-42.

[10] T. Körpınar, E. Turhan, Parallel Surfaces to Normal Ruled Surfaces of General Helices in the Sol Space Sol ${ }^{3}$, Bol. Soc. Paran. Mat., 2 (2013), 245-253.

[11] T. Körpınar, New type surfaces in terms of B-Smarandache Curves in Sol ${ }^{3}$, Acta Scientiarum Technology , 37 (2015), $389-393$.

[12] R. Lopez, Surfaces of constant Gaussian curvature in Lorentz-Minkowski Three- Space, Rocky Mountain Journal of Mathematics, 33 (2013), 971-993.

[13] R. Lopez, Parabolic surfaces in hyperbolic space with constant Gaussian curvature,

[14] E. Turhan, G. Altay, Minimal surfaces in three dimensional Lorentzian Heisenberg group, Beiträge zur Algebra und Geometrie / Contributions to Algebra and Geometry, DOI 10.1007/s13366-013-0156-4, 55 (2014), 1- 23.

[15] V. Velickovic, On Surfaces of Rotation of a given Constant Gaussian Curvature and Their Visualization,

[16] Z. Soyuçok, The Problem of Non- Trivial Isometries of Surfaces Preserving Principal Curvatures, Journal of Geometry, 52 (1995), 173- 188. 